\title{
Tracking medical 3D data with a parametric deformable model
}

\author{
Eric BARDINET ${ }^{1}$, Laurent D. COHEN ${ }^{2}$, Nicholas AYACHE ${ }^{1}$ \\ ${ }^{1}$ INRIA, B.P. 93 - 06902 Sophia Antipolis CEDEX, France. \\ ${ }^{2}$ CEREMADE, U.R.A. CNRS 749, Université Paris IX - Dauphine, France. \\ Email: bard@sophia.inria.fr
}

\begin{abstract}
This paper describes a method to fit and track surfaces. We suppose that we have already extracted from a 3-D image some data defined by a set of points. The fitting step makes use of a superquadric model and solves an inverse problem for free-form deformations. This is then used for tracking surfaces in a time sequence of $3 D$ images. We present different approaches to track surfaces in a sequence of $3 D$ images. From the tracking, we deduce a estimation of motion and visualize a velocity map. This method is applied to 2 sequences of different kinds of medical images to track the cardiac left ventricle.
\end{abstract}

\section{Introduction}

The analysis of cardiac deformations has given rise to a large amount of research in medical image understanding. Indeed, cardiovascular diseases are the first cause of mortality in developed countries. Various imaging techniques make it possible to get dynamic sequences of $3 \mathrm{D}$ images, that is $3 \mathrm{D}+\mathrm{T}$. The temporal resolution of these techniques is good enough to obtain a sufficient number of images between the muscle contraction (systole) and dilatation (diastole). These images are perfectly adapted to study the behavior of the cardiac system since they visualize how the heart walls deform. Processing these images opens numerous fields of applications, like the detection and analysis of pathologies.

Since it is characteristic of the good health of the heart, the left ventricle motion and deformation has been extensively studied by medical image processing groups as well as hospitals. Since its creation in 1989, our group has pioneered work in the use of deformable models to extract the left ventricle $([4,2])$. Other groups as well have also made various contributions to understanding the complex deformation of the ventricle $[6,7,5]$.

Over the last ten years, many surface reconstruction problems have been formulated as the minimization of an energy function corresponding to a model of the surface. Although previous approaches based on general deformable surfaces [4] give satisfactory results, they involve large linear systems to solve and heavy structures. This is why the parametric model presented here works better when dealing with a huge amount of data like a sequence of $3 \mathrm{D}$ images. The advantage of parametric deformable templates like superquadrics or hyperquadrics is their small number of parameters to represent a shape. However, if superquadric shapes give a good global approximation to a surface, the set of shapes described by superquadrics is too limited to approximate precisely complex surfaces. In [3], an implicit approach is used to refine the initial approximation. In a previous paper [1], we introduced a deformable superquadric model based on a superquadric fit followed by a Free Form Deformation (FFD).

After a brief review of the parametric model in section 2 , we present in section 3 different approaches to use this model to track surfaces in a sequence of $3 \mathrm{D}$ images. We then show in section 4 experimental results for an efficient tracking of the deformation of the left ventricle in different kinds of 3D medical images.

\section{Parametric Model to Fit unstruc- trured 3D data points}

In this section, we sketch the deformable model that we use for efficient tracking of the cardiac left ventricle. For more details and references on the complete algorithm, see [1]. First we fit 3D data with a superellipsoid, and then we refine this crude approximation using Free Form Deformations (FFD).

\subsection{Fit 3D data with superquadrics}

Superquadrics form a family of implicit surfaces obtained by extension of conventional quadrics. Superellipsoids are defined by the implicit equation:

$$
\left(\left(\left(\frac{x}{a_{1}}\right)^{\frac{2}{\epsilon_{2}}}+\left(\frac{y}{a_{2}}\right)^{\frac{2}{\epsilon_{2}}}\right)^{\frac{\epsilon_{2}}{\epsilon_{1}}}+\left(\frac{z}{a_{3}}\right)^{\frac{2}{\epsilon_{1}}}\right)^{\frac{\epsilon_{1}}{2}}=1 .
$$

Suppose that the data we want to fit with the superellipsoid are a set of $3 \mathrm{D}$ points $\left(x_{d}, y_{d}, z_{d}\right), i=1, \cdots, N$. We seek for the minimum of the following energy:

$$
E(A)=\sum_{i=1}^{N}\left[1-F\left(x_{d}, y_{d}, z_{d}, a_{1}, a_{2}, a_{3}, \epsilon_{1}, \epsilon_{2}\right)\right]^{2},
$$


where $F$ is the function defined by equation (1).

\subsection{Refinement of the fit with FFD}

We now have a parametric representation of the $3 \mathrm{D}$ data. We have to refine this representation. We use a global volumetric deformation called FFD. This is a tool devoted to the deformation of solid geometric models in a free-form manner. The main interest of FFD is that the resulting deformation of the object is just defined by a small number of points. This typical feature allows us to represent voluminous $3 \mathrm{D}$ data by models defined by a small number of parameters.

\subsection{Definition of FFD}

As an analogy, to understand FFD, consider a rubber box in which the object we want to deform is embedded. Control points are disposed on a regular volumetric grid in the box. The object follows in an way similar to a piece of flexible plastic the volumetric deformation of the box. The object points are linked to the box by: $\boldsymbol{X}=\boldsymbol{B} \boldsymbol{P}$, where $\boldsymbol{B}$ is the deformation matrix $N D \times N P(N D$ : number of points on the superellipsoid, $N P$ : number of control points), $\boldsymbol{P}$ is a matrix $N P \times 3$ which contains coordinates of the control points $\boldsymbol{P}_{\boldsymbol{i j k}}$, and $\boldsymbol{X}$ is a matrix $N D \times 3$ with coordinates of the model points. The elements of $\boldsymbol{B}$ are made of the tensor product of trivariate Bernstein polynomials. The deformation of the object is specified by moving the control points $\boldsymbol{P}_{\boldsymbol{i} \boldsymbol{j} \boldsymbol{k}}$ from their lattice positions. The position of a point of the deformed object is then computed with the previous relation.

\subsection{The inverse problem}

We use FFD to refine the representation of the data with the superellipsoid. Therefore we need to solve the inverse problem: first compute a displacement field between the superellipsoid and the data, and then, after having put the superellipsoid in a 3D box, search the deformation of this box which will best minimize the displacement field.

\subsection{Iterative algorithm}

To represent $3 \mathrm{D}$ data with the previous parametric deformable model, we use an iterative two-step algorithm:

- Let $\boldsymbol{P}_{0}$ be the parallelepiped box of control points; $\boldsymbol{X}_{0}=\boldsymbol{B} \boldsymbol{P}_{0}$ represents the set of points of the initial superellipsoid.

- We iterate the following two steps:

Step 1 : Computation of the displacement field $\delta \boldsymbol{X}_{n}$ such as: $\boldsymbol{X}_{n}^{a}=\boldsymbol{X}_{n}+\delta \boldsymbol{X}_{n}$

Step 2: Computation of the control points $\boldsymbol{P}_{n+1}$ by minimization of $\left\|\boldsymbol{B} \boldsymbol{P}-\boldsymbol{X}_{n}^{a}\right\|^{2}$

Computation of the deformed model:

$\boldsymbol{X}_{n+1}=\boldsymbol{B} \boldsymbol{P}_{n+1}$

Stop test: least-squares error $\left\|\boldsymbol{X}_{n+1}-\boldsymbol{X}_{n}\right\|$

An essential feature of this approach is that we use a volumetric deformation to deform a surfacic shape.
Figure $1: \boldsymbol{A}$ is a detailled example of the complete algorithm. In practice, we use boxes of size $5 \times 5 \times 5$ with data composed of around 6.000 points. So we get a compression ratio of 50 . Results are presented in figure $1: B$.

\section{Dynamic Tracking of the ventricle with the Parametric Model}

The parametric deformable model presented in the previous section allows us to represent a large number of unstructured 3D data points by a small number of parameters. On cardiac images, we obtain a compression rate of about 50 [1]. We now apply this model to track the left ventricle in various kinds of cardiac images. We first explain the chosen method to analyse a time sequence and then show examples on 2 sequences. Finally we use the parametric representation to infer quantitative information on the deformation.

\subsection{Dealing with a time sequence}

General deformable models usually need an initialization that is close enough to the solution. This is well suited for tracking in medical images since the deformation between two images is small and the model can start with the solution in the previous image as initialization for the current one. Deformable contours have been used for tracking boundaries since their introduction by Kass, Witkin and Terzopoulos [8]. This was applied to spatial tracking of ventricle boundary in successive cross sections of a $3 \mathrm{D}$ MR image in [4], in view of $3 \mathrm{D}$ reconstruction from a sequence of $2 \mathrm{D}$ models. With our parametric deformable model, the initialization is made automatically through the superquadric fit (see section 2.1), and then refined by the FFD. It is thus possible to make the reconstruction of each data set independently. However, having a previous refined model permits us to get increasing precision in the reconstruction. This leads to three possible approaches for tracking that are presented in figure 2 .

\subsection{Independent Representation}

This first approach consists of applying to each 3D image the complete model described so far. The advantage is that to define the model at time $n$, we do not need previous model information but only the superellipsoid and the control point box for this data. However this approach does not make use of the fact that the result at time $n$ is close to the one already computed at time $n-1$. This means that there is no temporal processing but a successive computation of static frames.

\subsection{Recursive Representation}

This method is a real temporal tracking. The complete model is applied only to the data of the first image, and then for time $n$, the model is obtained from the one at time $n-1$. This means that the shape obtained at time $n-1$ is itself put into a control point box instead of a superellipsoid in section 2.2. It results that the surface at time $n$ is obtained from the superellipsoid at time 1 iteratively deformed by the sequence of the $n$ first control point boxes. This has the advantage of being more and more precise when 
time increases since an accumulation of boxes allows the reconstruction of more complex shapes. However, since all previous boxes are needed to reconstruct the data at time $n$, this may be a difficulty when dealing with a long sequence of images.

\subsection{Independent Representation with a reference deformation}

The third approach is a trade-off between the two previous ones. The complete model is applied only to the data of the first image, and then for time $n$, the model is obtained from the one at time 1 . This means that the first reconstruction at time 1 is considered as a reference deformation of the superellipsoid. At time $n$, this reference shape is put into a control point box like in section 2.2 . It results that the surface at time $n$ is obtained from the superellipsoid at time 1 , followed by two deformations defined by the reference control point box and the current box. This has the advantage of both previous approaches. The approximation is more precise, being the iteration of two boxes and each data set can be retrieved from only one box and the first box and superellipsoid parameters. This is thus independent of the length of the time sequence. Since in practical applications, this method is as precise as the second one (using the iterative effect of $n$ boxes), as shown in figure 3 , this is the one we have chosen for the results presented in the next section.

\section{Application to the LV Wall Tracking in spatio-temporal data $3 \mathrm{D}+\mathrm{T}$}

We present in this section applications of the algorithm on 3D medical data stemming from cardiac imagery. The data have been obtained from 2 kinds of acquisition: nuclear medecine (SPECT image) and volumetric X-ray scanner (Dynamic Spatial Reconstructor - Mayo Clinic). A first problem to deal with is the segmentation of the data, in order to get a set of $3 \mathrm{D}$ points which corresponds to the structure we want to study (the cardiac left ventricle). We used morphological operators to segment the images, and extract an isosurface from the resulting binary images to get the sets of $3 \mathrm{D}$ points that we need for the reconstruction algorithm. The SPECT MIBI image is a volume of $64 \times 64 \times 64$ voxels. The DSR image is an image with very good resolution $(98 \times 100 \times 110$ for a voxel of $0.926 \mathrm{~mm}^{3}$ ).

We present the results of tracking of the left ventricle in 2 time sequences (successive time frames in a cardiac cycle) of $3 \mathrm{D}$ images:

Nuclear medicine data (SPECT MIBI) This sequence is composed of 8 frames. Figure $4: \boldsymbol{A}$ shows the dynamic sequence on a cross section, with the segmented and reconstructed surfaces superimposed on the grey level image. The model is defined by a $5 \times 5 \times 5$ box. Figure $4: B$ represents the same result for $3 \mathrm{D}$ rendering of the surface.

Scanner data (DSR) This sequence is composed of 18 time frames. Figure 5 shows the dynamic sequence on a cross section, with the segmented epicardium and endocardium surfaces superimposed on the grey level image. It also shows the reconstruction of these two surfaces using either one or two models. Using only one model means that the two surfaces are put in a same control points box, and the minimisation of equation (2.3) is done simultaneously on the union of both displacement fields. This shows that our model can handle the deformation of many connected components at the same time by merging the respective displacement constraints. This makes no difficulty since we have a $3 \mathrm{D}$ box which defines a really $3 \mathrm{D}$ deformation.

\subsection{Motion}

The reconstruction and representation of a time sequence of surfaces by a sequence of parametric models permits one to visualize the estimation of the deformation in time. This flow field can then be used to extract characteristic parameters and give a diagnostic interpretation for the patient. Such a set of parameters is obtained in [6] to quantify the left ventricle deformation. These parameters are similar to those used by the cardiologist for diagnosis. With the same goal in mind, we use our sequence of models to extract the time trajectory of each point of the surface during a cardiac cycle. The approximation we make is that for a point in a frame, its position in the next frame is the closest point to the current data. This hypothesis is valid in the case of small deformation, which means that the time step is not too large. Note also that the initial superellipsoid match allows us to have a global registration which is then refined.

\subsubsection{Trajectories}

Listing the successive positions of a point of the reconstructed surface model along the time sequence, we obtain the trajectory followed by this point. Figure 6 shows the trajectories of the node points between the diastole and the systole. These are represented on four different viewpoints to appreciate better the motion. It can be seen, especially on the upper and lower views, that the model makes explicit the characteristic twist component of the motion. This torsion could be quantified to detect some pathologies of the ventricle.

\subsubsection{Range of the displacements}

The pointwise tracking of the deformation permits to give an evaluation of the velocity field during the sequence. The visualization of these displacements by different colors, according to their range, on the surface shows up clearly areas on the ventricle where the deformation is weak. This vizualisation could be used to allow the localization of pathologies like infarcted regions.

\section{Conclusion}

We presented a new approach to shape reconstruction and tracking applied to $3-\mathrm{D}$ medical data. It is based on a parametric deformable model that give a compact representation of a set of points in a $3-\mathrm{D}$ image, by reconstruction of the displacement field between the data and model. Three approaches were presented to use this model in order to track efficiently the left ventricle wall in a sequence of 3D images during a cardiac cycle. The deformation field extracted 
permits to visualize clearly of high and low displacements. This could be used to detect infarcted areas. Experimental results have been shown for automatic shape tracking in time sequences of 2 kinds of medical images.

\section{Acknowledgements}

We would like to thank Grégoire Malandain for fruitful discussions about segmentation, Jérôme Declerck, Serge Benayoun and Alexis Gourdon for constructive remarks, and also Mike Brady for his judicious advices. This work was partially supported by Digital Equipment Corporation.

\section{References}

[1] E. Bardinet, L.D. Cohen, and N. Ayache. Superquadrics and free-form deformations: a global model to fit and track 3d medical data. In International Conference on Computer Vision, Virtual Reality and Robotics in Medecine (CVRMed'95), Nice, France, April 1995.

[2] S. Benayoun, C. Nastar, and N. Ayache. Dense nonrigid motion estimation in sequences of $3 \mathrm{~d}$ images using differential constraints. In International Conference on Computer Vision, Virtual Reality and Robotics in Medecine (CVRMed'95), pages 309-318, Nice, France, April 1995.

[3] I. Cohen and L.D. Cohen. A hybrid hyperquadric model for 2-D and 3-D data fitting. In Proceedings of the 12th IEEE International Conference on Pattern Recognition (ICPR'94), pages B-403-405, Jerusalem, 1994. Part of Inria TR 2188, to appear in Computer Vision, Graphics, and Image Processing : Image Understanding.

[4] L.D. Cohen and I. Cohen. Finite element methods for active contour models and balloons for $2-\mathrm{D}$ and $3-\mathrm{D}$ images. IEEE Transactions on Pattern Analysis and Machine Intelligence, PAMI-15(11), November 1993.

[5] T. McInerney and D. Terzopoulos. A dynamic finite element surface model for segmentation and tracking in multidimensionnal medical images with application to cardiac 4d image analysis. Computerized Medical Imaging and Graphics, 19:69-83, January 1995.

[6] J. Park, D. Metaxas, and A. Young. Deformable models with parameter functions : application to heartwall modeling. In IEEE Computer Vision and Pattern Recognition, pages 437-442, June 1994.

[7] P. Shi, A. Amini, G. Robinson, A. Sinusas, C. Constable, and J. Duncan. Shape-based 4d left ventricular myocardial function analysis. In IEEE Workshop on Biomedical Image Analysis, pages 88-97, Seattle, June 1994.

[8] D. Terzopoulos, A. Witkin, and M. Kass. Symmetryseeking models for $3 \mathrm{D}$ object reconstruction. International Journal of Computer Vision, 1(3):211-221, October 1987.

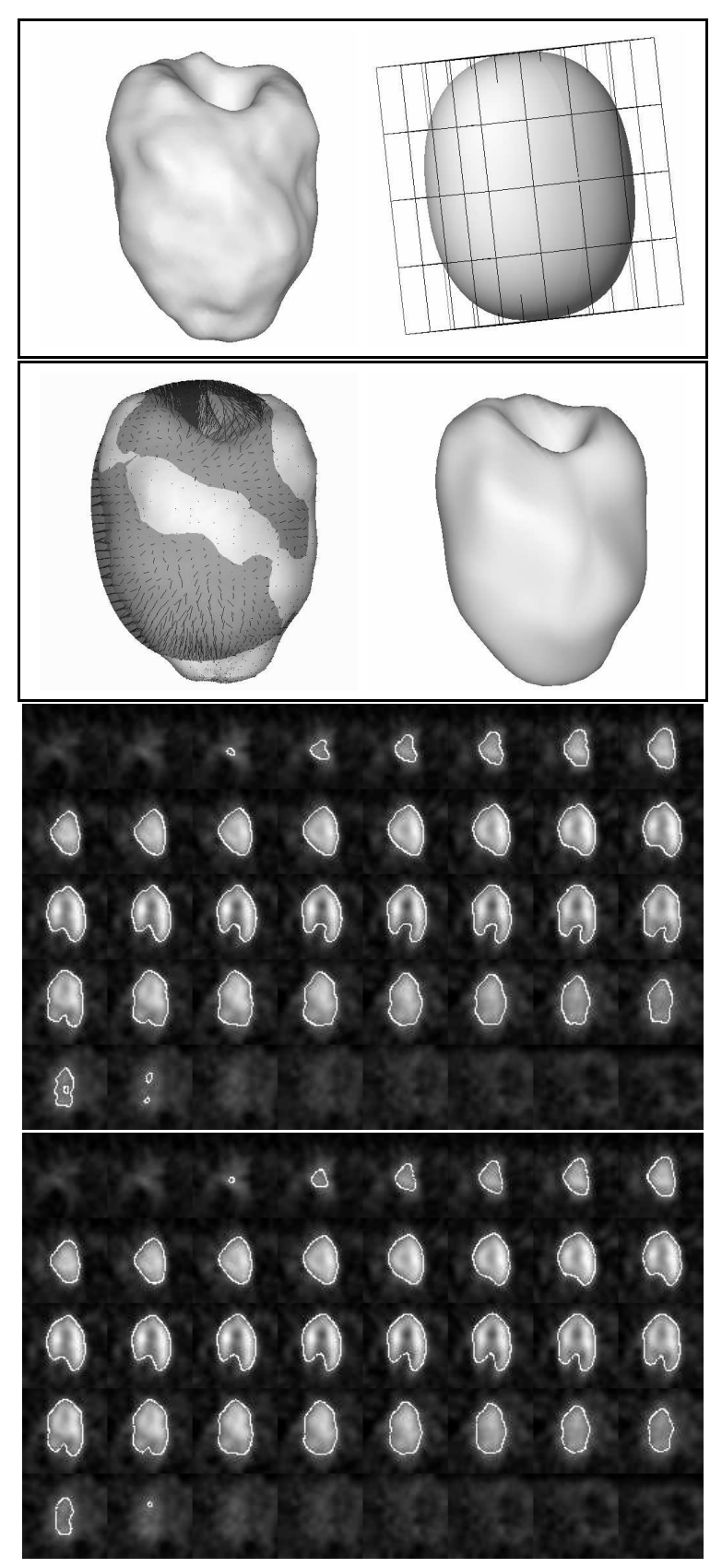

Figure 1: $\boldsymbol{A}$ : From the superellipsoid to the final model. Top left: data. Top right: superellipsoid fit and initial box of control points. Bottom left: displacement field between data and the superellipsoid. Bottom right: final model after minimization of the displacement field. $\boldsymbol{B}$ : Segmentation and representation of the epicardium (external wall of the ventricle) for the 3D image of the left ventricle (SPECT MIBI). Top: isosurface superimposed to the image. Bottom: reconstruction by the model. 


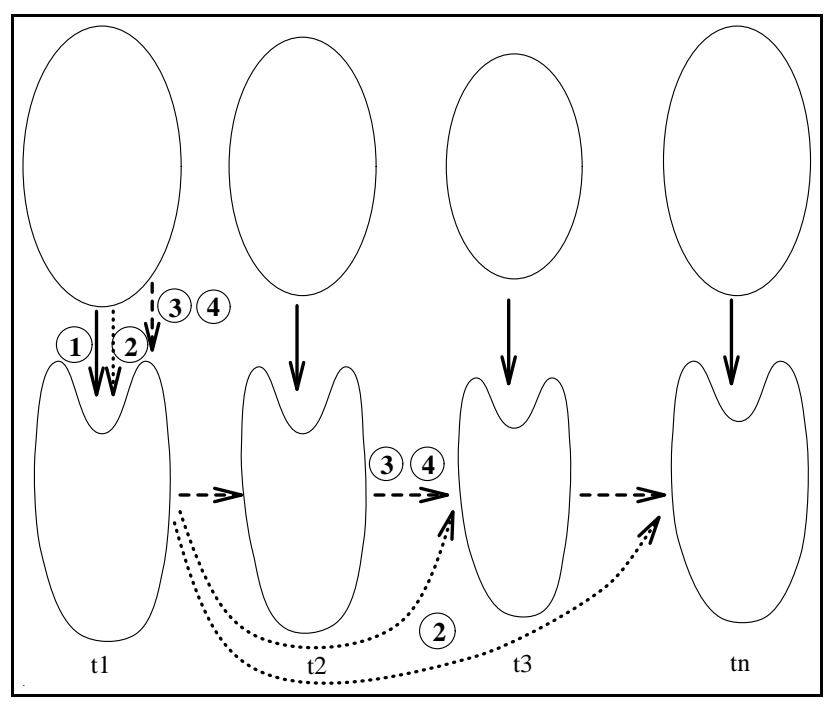

Figure 2: Three different approaches to deal with a temporal sequence. 1 : Data reconstruction at each time step using the superquadric and FFD fit. 2 : Data reconstruction at time step $n$ using only the FFD from the model found at time $n-1$. 3 : Data reconstruction at time step $n$ using only the FFD from the model found at time 1 .

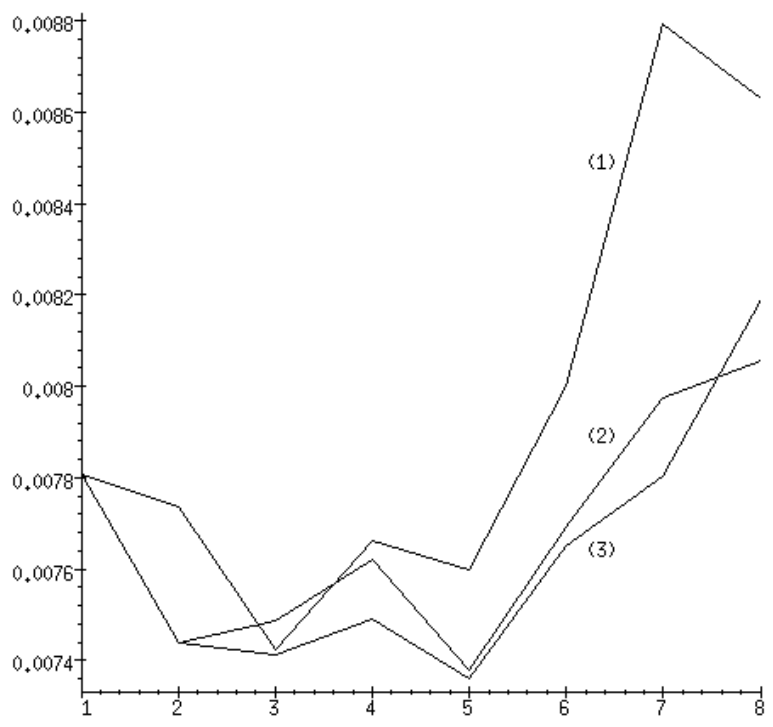

Figure 3: Dealing with a time sequence. Time evolution of the least square error between the data and model for 8 frames. The three curves correspond to the three approaches. The larger error is obtained with the approach 1.
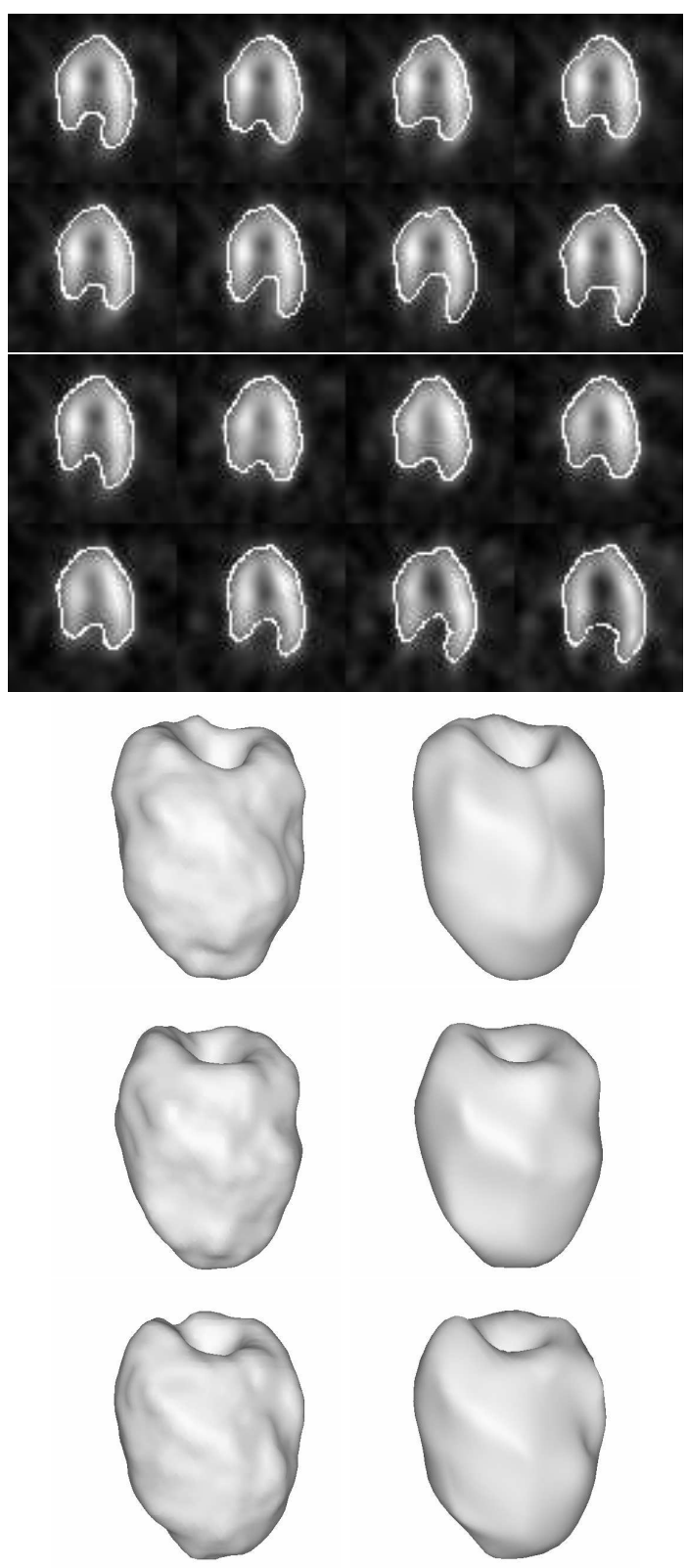

Figure 4: $\boldsymbol{A}$ : Segmentation and representation of the epicardium (external wall of the ventricle) for a time sequence $3 \mathrm{D}+\mathrm{T}$ of the left ventricle during the cardiac cycle (SPECT MIBI); visualisation of a cross section. Top : isosurface superimposed on the image. Bottom : reconstruction by the model. $\boldsymbol{B}$ : Time sequence of the epicardium. On the left : isosurface obtained by data segmentation SPECT MIBI (6000 points). On the right : representation by the parametric model (130 parameters). 


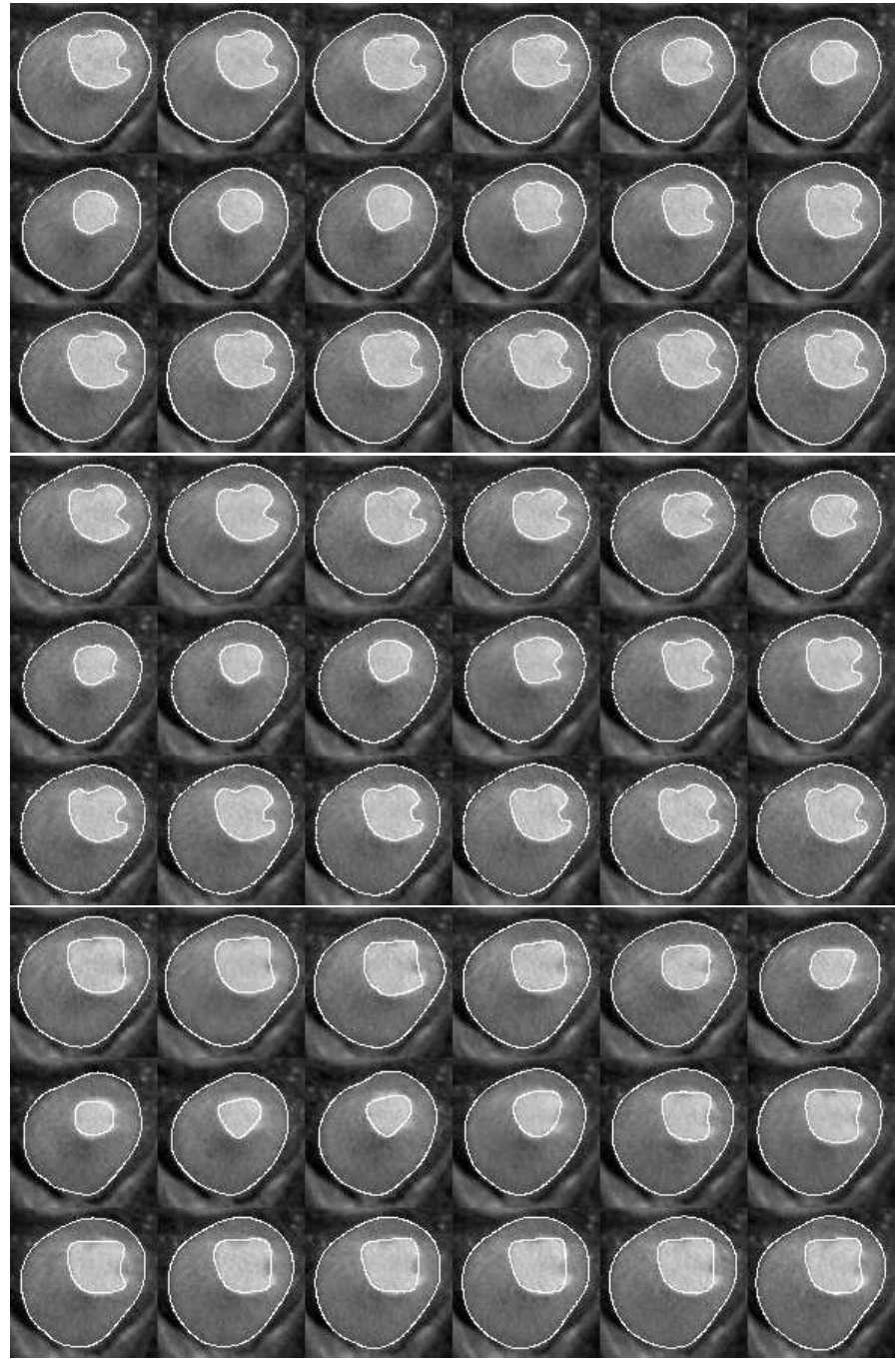

Figure 5: Segmentation and representation of the epicardium and endocardium for a time sequence $3 \mathrm{D}+\mathrm{T}$ of the left ventricle during the cardiac cycle (Scanner DSR); visualisation of a cross section. Top : the two isosurfaces superimposed on the image. Middle : reconstruction by two separate models. Bottom : simultaneous reconstruction of both surfaces by one model.
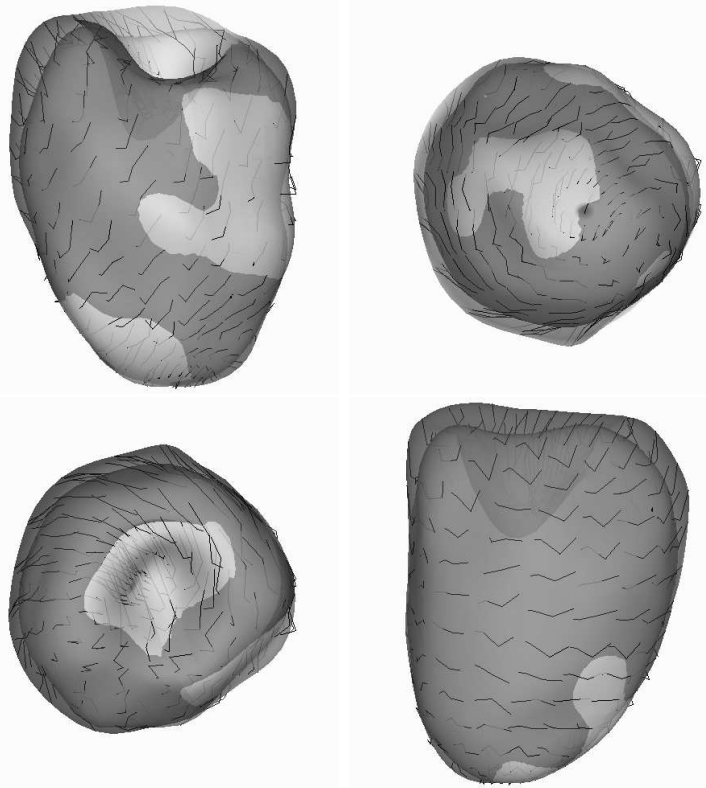

Figure 6: Trajectories of the model points during a cardiac cycle from 4 viewpoints. The two surfaces represent the models at end of diastole (dilatation) and systole (contraction).

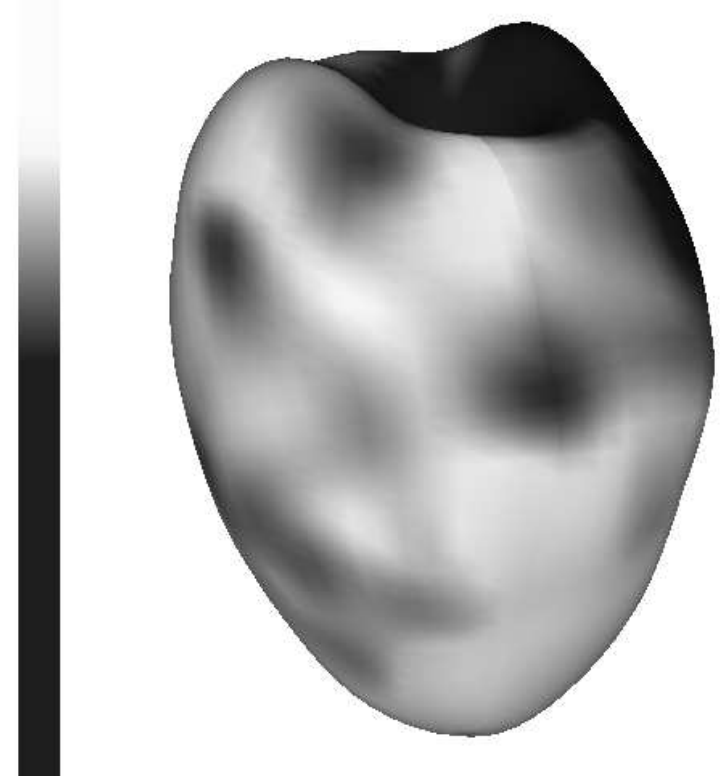

Figure 7: Range of the displacements of the model points during a cardiac cycle (light areas correspond to weak deformations). 